Simultaneous measurements of $S_{v}$ at 2,695 and $8,085 \mathrm{MHz}$ allow the determination of $T_{\mathrm{c}} \Omega_{\mathrm{s}}$ and $E T_{\mathrm{c}}^{-1.45}$ as functions of time whenever the argument in the exponential in equation 1 is large enough. By multiplying $E T_{c}^{-1.45}$ by $T_{c} \Omega_{\mathrm{s}}$ raised to the 1.45 power, a parameter independent of $T_{e}, E \Omega_{s}^{1.45}$ can be obtained. The results of such an analysis, whenever it was possible, are presented in Table 1. Only the first two hours on May 3 are not compatible with a thermal source model. We conc'xde from this that, just as Sco X-1 occasionally shows signs of a rare thermal component ${ }^{4}$, Cyg X-3 contains both thermal and non-thermal components, with the non-thermal behaviour being rare.

A few simple conclusions can be drawn from the thermal source parameters derived in Table 1. First, as has previously been noted for $\beta$ Persei (R. M. H., manuscript submitted), $T_{e} \Omega_{3}$ is roughly constant, except for variations as expected from the noise in the flux density data, on any particular day; for example, $T_{\mathrm{c}} \Omega_{\mathrm{s}}=1.9 \times 10^{-6} \mathrm{~K} \mathrm{sr}$ on May $1,1.0 \times 10^{-6}$ on May 2, and $2.6 \times 10^{-6}$ on May 3. The most interesting differences occur in $E \Omega_{s}^{1.43}$ which was roughly constant at $7.0 \times 10^{-8}$ on May 1 and $1.1 \times 10^{-7}$ on May 3 ; however, there was a clear variation from $5 \times 10^{-8}$ up to $1.6 \times 10^{-7}$ during the flarc on May 2, 1972. If the thermal interpretation of the radio data for Cyg X-3 is valid, simultaneous measurements at radio and $\mathrm{X}$-ray energies should provide an unusual opportunity to look for correlations and to further determine the parameters of the source.

We thank Giacconi et al. ${ }^{2}$ for providing a copy of the UHURU X-ray source catalogue in advance of publication. The National Radio Astronomy Observatory is operated by Associated Universities Inc. under contract with the US National Science Foundation.

R. M. Huellming

M. HERMANN

E. WeBster

National Radio Astronomy Observatory.

Green Bank,

West Virginia

Received May 31, 1972.

1 Braes, L. L. E., and Miley, G. K., Nature, 237, 506 (1972).

2 Giacconi, R., Murray, S., Gursky, H., Kellogg, E., Schreier, E., and Tananbaum, H., Astrophys. J. (in the press).

3 Hjellming, R. M. and Wade, C. M., Astrophys. J., 164, L1 (1971).

4 Wade, C. M., and Hjellming, R. M., Astrophys. J., 170, 532 (1971).

s Wade, C. M., and Hjellming, R. M., Nature, 235, 270 (1972).

6 Hjellming, R. M., Wade, C. M., and Webster, E., Nature, 236, 43 (1972).

\section{LR Cen is not Cen X-3}

THE eclipsing variable LR Cen has been suggested ${ }^{1,2}$ as a candidate for the optical counterpart of $\mathrm{Cen} X-3^{3}$ on the basis of the near coincidence of the position and period of LR Cen with those of Cen X-3. My observations ${ }^{4}$ and those of Kristian et al. ${ }^{1}$ show that in March, the time of eclipse of the secondary of LR Cen nearly coincided with that of Cen X-3. Also LR Cen and Cen $\mathrm{X}-3$ both have orbits of low eccentricity; thus, LR Cen and Cen $X-3$ have similar positions, periods, phases and eccentricities.

The difference in periods, however, though small, is significant. The observed eclipses of the X-ray source would have occurred at several different phases in the cycle of LR Cen if its period was unchanged since the original determination of 2.095595 \pm 0.000009 days by Uitterdijk ${ }^{5}$ in 1932 . I have redetermined the period of LR Cen from my observations of its primary eclipse on March 16,1972 (Fig. 1a) and from those obtained on April 26, 1972 (Fig. 1b). The precise times of the minima were determined from the coefficients of second order polynomials which were fitted to the observations using the method of least squares. The value for the period of LR Cen of $2.0954 \pm 0.0007$

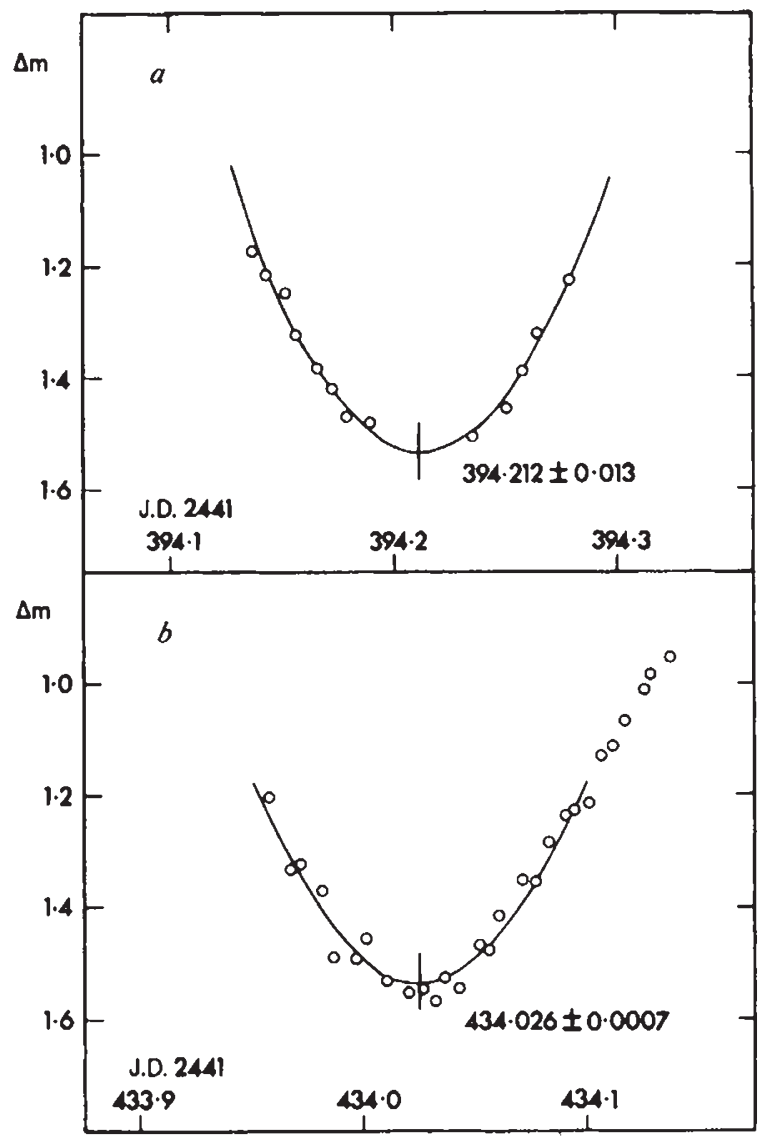

Fig, 1 Primary minima of LR Cen in V, given in magnitudes with respect to Uitterdijk's comparison star $C$. The lines represent the second order polynomials used to determine the time of the minima.

days was obtained for the 19 cycles elapsed between the primary minimum on March 16, 1972 (J.D. $2441394.212 \pm 0.013$ ) and the primary minimum on April 26, 1972 (J.D. 2441434.026 \pm 0.007 ). These observations show that the period of LR Cen is unchanged, and that it is significantly different from 2.08712 \pm 0.00004 days, the period of Cen X-3.

Thus, the near coincidence of the times of eclipse found in March is wholly coincidental, and I now conclude that LR Cen is not the optical counterpart of Cen X-3.

Bruce A. Peterson

Mount Stromlo and Siding Spring Observatories,

Research School of Physical Sciences,

The Australian National University

Received May 30, 1972.

1 Kristian, J., Brucato, R. J., and Westphal, J. A., Astrophys, J. Lett. (in the press)

2 Shklovsky, J. S., Cherepashchuk, A. M., and Efremov, Y. N., Nature, 236, 448 (1972).

${ }^{3}$ Schreier, Ė, Levinson, R., Gursky, N., Kellogg, E., Tananbaum, H., and Giacconi, R., Astrophys. J. Lett., 172, L79 (1972).

4 Peterson, B. A., Nature, 236, 449 (1972).

5 Uitterdijk, F., Bull. Astron. Insts. Netherlands, 6, 295 (1932).

\section{Ring Current Effect on Magnetospheric Electron Density Profiles derived from Plasmapause Whistlers}

THE longitudinal drift of van Allen radiation belt charged particles, with opposite charges drifting in opposite directions in the geomagnetic field, constitutes a westward directed ring 